\title{
Pancreatic cancer: Clinical and basic research
}

With its incidence (10-11 per 100, 000 people) almost equaling its prevalence, pancreatic cancer is one of the most aggressive human tumors. Understanding the exceptionally aggressive behavior has been the objective of clinical and basic research elucidating in detail the molecular changes that lead to the development and the progression of this disease. The molecular alterations observed range from gross chromosomal abnormalities to gene mutations and epigenetic changes, which contribute together to the aggressive growth of pancreatic cancer with retroperitoneal and perineural infiltration, angioinvasion, and early metastases. Clinically, pancreatic cancer is characterized by high rates of local relapse after resection, and resistance to most of the available treatment regimens, making patient management a complex and challenging task.

Surgery: Curative resection is the single most important factor determining the outcome in patients with pancreatic cancer. Mortality and morbidity rates inversely correlate with the number of pancreatic tumor resections per year. Thus, in high volume centers, resection-related mortality and morbidity have substantially decreased over the past decades, and mortality rates less than $5 \%$ have been achieved. In addition, the long-term survival of patients who undergo pancreatic resection in high-volume centers is superior as well, making an even stronger case for centralization of pancreatic surgery. The pylorus-preserving partial pancreaticoduodenectomy offers long-term survival, quality of life and weight gain identical to those of the classical Whipple procedure. Extended lymphadenectomy with retroperitoneal clearance has not resulted in superior outcome and should not be performed outside of clinical studies.

Adjuvant therapy: Adjuvant chemotherapy with 5-FU/ FA according to the ESPAC-1 protocol is the current standard, whereas adjuvant chemoradiation does not seem to offer a survival benefit. Nonetheless, encouraging results with an interferon-based adjuvant chemoradiation protocol have been reported with a 5-year survival rate of $55 \%$. This study provides hope for a substantial increase

Correspondence to: Helmut Friess, M.D., Department of General Surgery, University of Heidelberg, Im Neuenheimer Feld 110, 69120 Heidelberg, Germany. Email: helmut_friess@med.uni-heidelberg.de in survival and quality of life following pancreatic cancer resection, but needs further confirmation in randomized controlled trials.

Palliative therapy: In non-resectable and/or metastatic pancreatic cancer, the mainstay therapy is monotherapy with gemcitabine, despite proven low effectiveness. So far, classic cytotoxic agents, such as 5-fluorouracil, pemetrexed, irinotecan, exatecan, cisplatin, and others, given alone or in combination with gemcitabine, have not proven to be superior. However, results of phase III studies with the combination of gemcitabine with oxaliplatin or erlotinib (Tarceva ${ }^{\mathrm{TM}}$ ) seem promising.

Outlook: Recent advances in our understanding of the molecular behavior of pancreatic cancer and the subsequent implementation of clinical studies promise improved five-year survival rates for at least a subgroup of patients. For example, several of the identified molecular alterations of pancreatic cancer have been studied as possible therapeutic targets. One of the most promising molecular system for drug development is the EGF receptor (EGFR)/ligand cascade, which triggers not only mitogenic and anti-apoptotic responses but also cell migration. For example, the EGFR tyrosine kinase inhibitor gefitinib (Iressa ${ }^{\mathrm{TM}}$ ) negatively influences pancreatic cancer cell growth, invasion and colony formation. First results with blockage of the EGFR by gefitinib, erlotinib, and cetuximab (Erbitux ${ }^{\mathrm{TM}}$ ) in phase I-III clinical trials underline the important role of EGFR signaling in pancreatic cancer. Further progress will be achieved by combining earlier diagnostic markers, surgery and chemotherapy/radiotherapy with targeted molecular and immune therapies. Therefore, a main focus of academic medicine in the field of pancreatic cancer will be translational research, transferring advances from basic research to clinical studies for the benefit of pancreatic cancer patients.

Leading clinical and basic researchers from China and Germany present in this special issue on pancreatic cancer of the Chinese-German Journal of Clinical Oncology the state-of-the-art in diagnostic, surgery and therapy of pancreatic cancer. We believe that this thematic issue will provide an interesting update and look into the future for the management of pancreatic cancer. 